\title{
Wideband Dual-Polarized Patch Antenna with Capacitive Coupling for mm-Wave Bands
}

\author{
Marko Sonkki ${ }^{1}$, Danping $\mathrm{He}^{2}$, Zeeshan Siddiqui ${ }^{1}$, Marko E Leinonen ${ }^{1}$, Ke Guan ${ }^{2}$ \\ ${ }^{1}$ Centre for Wireless Communications (CWC), University of Oulu, Oulu, Finland, marko.sonkki@gmail.com \\ ${ }^{2}$ State Key Laboratory of Rail Traffic Control and Safety, Beijing Jiaotong University, China, hedanping@bjtu.edu.cn
}

\begin{abstract}
This paper presents a planar wideband dualpolarized antenna structure integrated on PCB (Printed Circuit Board). The patch itself is fed by a capacitive coupling with two smaller patches, one for each polarization. Whereas the simulations predicts $24-40 \mathrm{GHz},-10 \mathrm{~dB}$ impedance bandwidth. The measured one shows $24.75-42.75 \mathrm{GHz}$ bandwidth. The results are corresponding to $50 \%$ relative $-10 \mathrm{~dB}$ impedance bandwidth. The patch antenna is on the ground plane of size $4.7 \mathrm{~mm} \times 4.7 \mathrm{~mm}$, and the corners of the ground plane are cut to gain better XPD (cross polarization discrimination). The manufactured prototype antenna is measured and simulated with a $50 \Omega$ coaxial line. Simulated total efficiency and XPD are presented as a function of frequency. The total efficiency is better than $-0.8 \mathrm{~dB}(83 \%)$ efficiency, whereas the simulated XPD is better than $14 \mathrm{~dB}$. The simulated 3D radiation patterns are presented at $24 \mathrm{GHz}$, $32 \mathrm{GHz}$, and $40 \mathrm{GHz}$ with gains of $2.8 \mathrm{dBi}, 5.0 \mathrm{dBi}$, and $4.3 \mathrm{dBi}$, respectively.
\end{abstract}

Index Terms-Antenna isolation, capacitive feed, cross polarization discrimination (XPD), mutual coupling, planar antenna, printed circuit board (PCB).

\section{INTRODUCTION}

Now, as fifth-generation $(5 \mathrm{G})$ wireless communication systems are coming to the commercial markets, they are expected to offer a 1000-fold high-speed wireless data transmission links with a low latency, compared with the

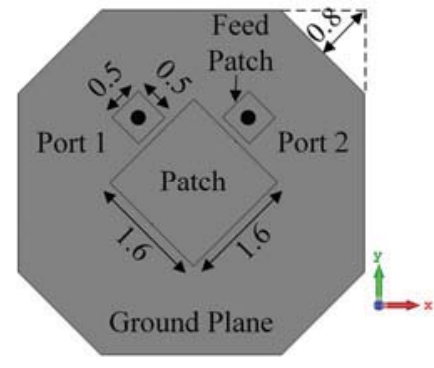

(a)

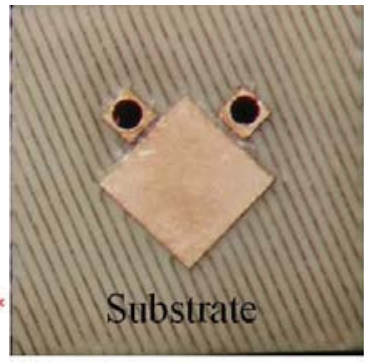

(b)

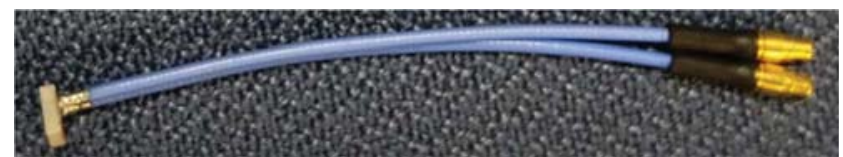

(c)

Fig. 1. (a) Wideband dual-polarized patch antenna structures with dimensions (in $\mathrm{mm}$ ) and coordinate system. Gray color corresponds with conducting material. (b) Prototype antenna from the top and (c) from the side. Notice that (b) and (c) are not in the same scale. earlier generation systems. 5G wireless system also offer a wider spectrum for higher data rates and this is possible by using frequency bands at higher frequencies. A rapid development in radio telecommunications systems must meet these requirements. Nowadays, the evolution of wireless access technologies are reaching these demands by using millimeter waves (mm-Wave) [1], where spectrum is highly attractive because of the available bandwidth [2][3].

In order to support multiple bands, especially for cellular applications, antennas with a wide frequency range can offer a good and alternative approach for an antenna design. Antennas should be not only easy to integrate with active and passive components but also fabricated by a simple manufacturing process to gain a low price. To integrate various wireless systems into a single device, components including antennas must be small to guarantee a compact integration without forgetting an optimum functionality.

Planar antenna structures are flexible and cost-effective candidate for many applications. Planar dipoles and bowtie structures can be wideband [4][5]. However, with dipole kind of structures, a balun is needed for operation, corresponding to a differential excitation. Related to the bow-tie-shaped antennas integrated on PCB (Printed Circuit Board) with ground plane, they are lately investigated for broadband applications perspective [6]-[9].

Patch antennas are also studied at mm-Waves, but wideband patch antennas are not so intensively studied, especially dual-polarized structures, and only few references can be found. A linearly polarized patch antenna with $37 \%$ fractional bandwidth (FBW) around $27 \mathrm{GHz}$ is demonstrated for mm-Waves applications in [10]. A substrate-integratedwaveguide (SIW) fed, linearly polarized antenna for $57-71 \mathrm{GHz}$ with $37 \% \mathrm{FBW}$, is demonstrated for $5 \mathrm{G}$ applications in [11]. In [12], a dual polarized, stacked patch antenna for mm-Wave base stations is presented with $14 \%$ FBW. Still, when thinking about the commercial wireless devices and base stations, a patch antenna is a low cost structure, easy to manufacture in industrial mass production processes.

This paper presents a wideband dual-polarized patch antenna structure for mm-Waves at $24-40 \mathrm{GHz}$ bandwidth. This corresponds to $50 \% \mathrm{FBW}$, with $-10 \mathrm{~dB}$ impedance bandwidth criteria. The simulated total efficiency is better than $-0.8 \mathrm{~dB}(83 \%)$, and the simulated isolation between the antenna ports is better than $13 \mathrm{~dB}$. The simulated XPD 


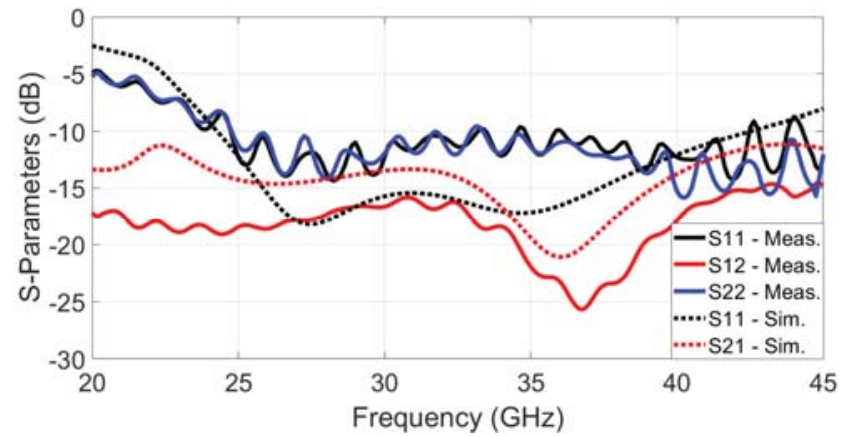

Fig. 2. Measured and simulated frequency response of the studied wideband dual-polarized patch antenna.

(Cross Polarization Discrimination) is better than $14 \mathrm{~dB}$. The antenna excitation is done with coaxial probes, which are connected to the coupling patches, suitable for measurement setup. In the authors' best knowledge, only few wideband dual-polarized patch antennas for $\mathrm{mm}$-Waves are presented in the literature.

\section{ANTENNA STRUCTURE}

This section presents the studied dual-polarized patch antenna structure, integrated as a PCB over a conductive ground plane. Fig. 1(a) presents antenna structure with the dimensions in millimeters, along with the coordinate system. The prototype antenna is based on the simulation model with $50 \Omega$ coaxial probe feed. The fabricated prototype antenna is presented in Fig. 1(b) and (c).

The antenna structure, or a unit cell, is designed for the radio channel measurements, by finding a compromise between the impedance matching, the isolation between the antenna excitation ports, and the XPD.

A coaxial probe feed with a waveguide port excitation is used to simulate the antenna. CST Microwave Studio is used in all the simulations. The size of the substrate is $4.7 \times 4.7 \mathrm{~mm}^{2}$, which is also the overall dimensions of the unit cell. These dimensions are corresponding to $75 \%$ improvement in area, and 59\% in volume, compared to our earlier contribution. [9]. Regarding the antenna geometry, the gap between the patch antenna and the feed patch is $100 \mu \mathrm{m}$. The RF-laminate used in this proposed study is Rogers 4003C with $1.524 \mathrm{~mm}$ substrate thickness and $\varepsilon=3.38$. The coaxial cables used in the prototype antenna have a length of $50 \mathrm{~mm}$ with $0.6 \mathrm{~dB}$ loss at the used cable length, and the connection interface is SMPM (Sub-Miniature Push-on Micro).

In terms of wavelength, the electric distance of the patch element from the conductive ground plane is between $0.22 \lambda-0.37 \lambda$, depending on the operating frequency (24-40 GHz). Thus, the theoretical optimum $\lambda / 4$ distance with the used laminate electric properties is at $28 \mathrm{GHz}$.

The corners of the ground plane are cut to find a good compromise between S-parameters and XPD over the studied bandwidth. The purpose is to keep XPD better than $10 \mathrm{~dB}$, and the isolation between antenna ports as good as possible. This is done by cutting $0.8 \mathrm{~mm}$ out from every

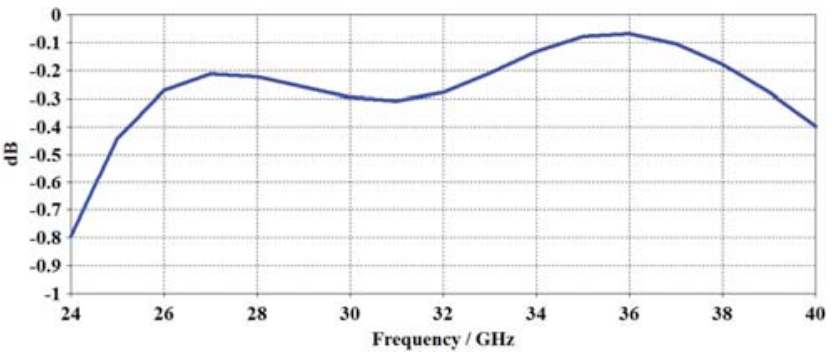

(a)

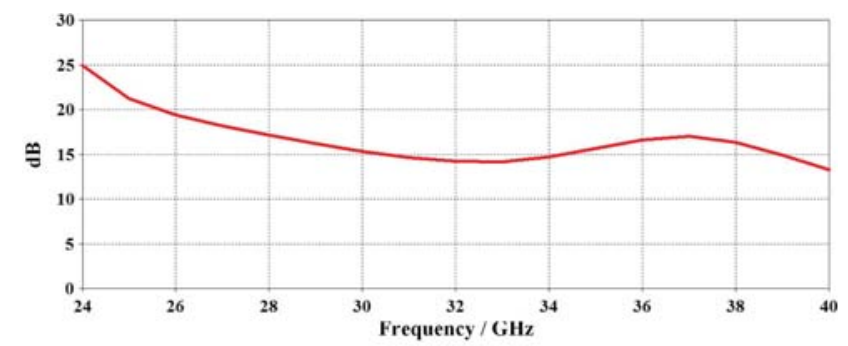

(b)

Fig. 3. Simulated (a) total efficiency and (b) XPD of the studied wideband dual-polarized patch antenna.

corner of the ground plane (see Fig. 1(a)), whereas the substrate shape has been kept as a square. The S-parameters are measured and compared to the simulated results in the next section.

\section{RESULTS}

This section presents the simulated and measured results of the studied wideband dual-polarized patch antenna structure, presented in the Section II. First, the antenna performance is studied in terms of simulated and measured impedance matching and mutual coupling between the antenna ports. Then, the simulated radiation properties are presented in terms of total efficiency, XPD, and radiation patterns at 24, 32, and $40 \mathrm{GHz}$.

\section{A. Simulated and Measured S-Parameters}

Simulated and measured S-parameters are presented in Fig. 2. The simulated results are predicting $24-40 \mathrm{GHz}$ $-10 \mathrm{~dB}$ impedance bandwidth with better than $13 \mathrm{~dB}$ isolation over the studied bandwidth. As the structure is symmetrical, only $S_{11}$ is presented. In measured results, the lower frequency has shifted slightly higher to $24.75 \mathrm{GHz}$, whereas higher frequency moved to $42.50 \mathrm{GHz}$. Thus, the measured $-10 \mathrm{~dB}$ impedance bandwidth is $24.75-42.50 \mathrm{GHz}$. This corresponds to $53 \%$ FBW when simulated predicted $50 \%$ FBW. Related to the measured isolation, the results shows better than $16 \mathrm{~dB}$ within the studied bandwidth.

As a conclusion, the studied wideband dual-polarized patch antenna shows that the measured results very well follow the simulated ones with a fair margin. The ripple seen in the measurement results are related to the cables, as they could not be calibrated out in the calibration process. This also have a slight effect to the impedance matching as the cable losses are approximately $0.6 \mathrm{~dB} / 5 \mathrm{~cm}$. 

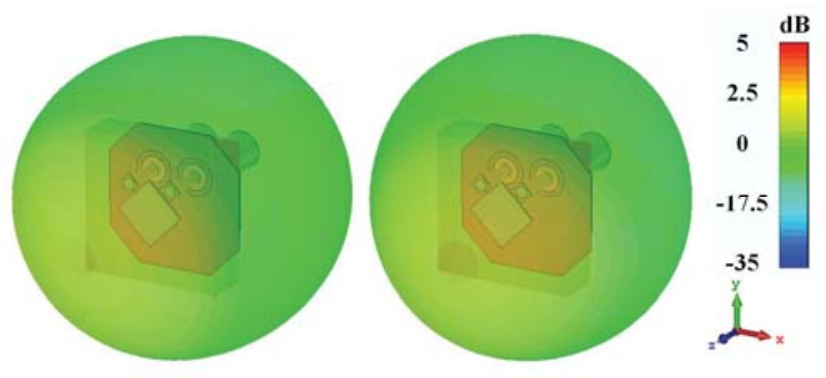

(a)
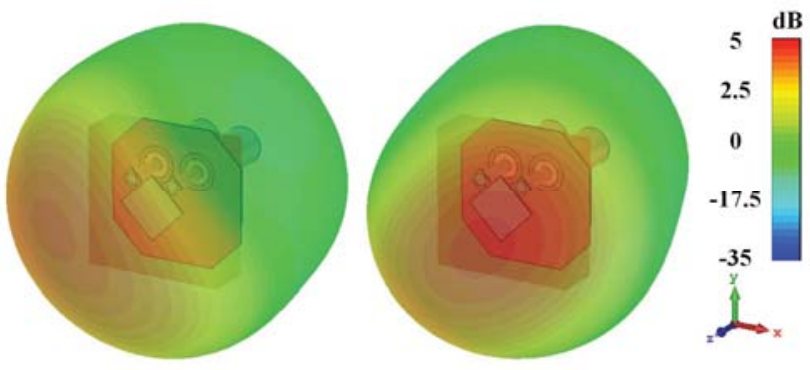

(b)
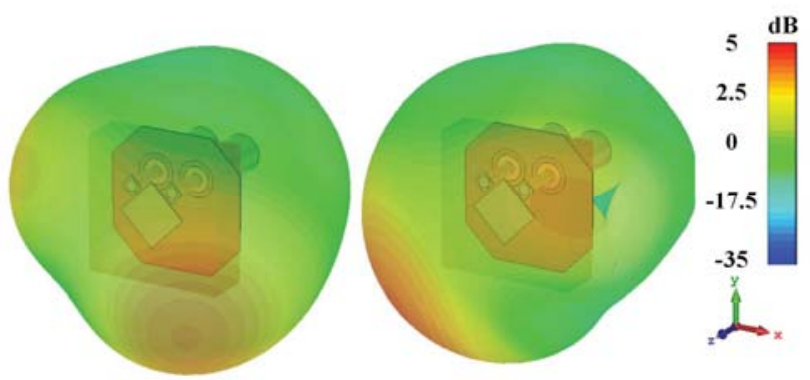

(c)

Fig. 4. The simulated 3D radiation patterns at (a) $24 \mathrm{GHz}$, (b) $32 \mathrm{GHz}$, and (c) $40 \mathrm{GHz}$. The left side presents radiation pattern of Port 1 and right side the Port 2.

\section{B. Simulated Radiation Properties}

Fig. 3(a) and (b) presents the simulated total efficiency and XPD of Port 1, respectively. As mentioned in the previous sub-section, because of the symmetry, only results of the one port is presented. The simulations predict better than $-0.8 \mathrm{~dB}(83 \%)$ the total efficiency over the studied $24-40 \mathrm{GHz}$ bandwidth. Related to the Fig. 3(b), the simulations predict better than $14 \mathrm{~dB}$ XPD over the studied bandwidth. XPD is calculated in the Z-direction (see Fig. 4).

Fig. 4 presents the simulated $3 \mathrm{D}$ radiation patterns of the studied antenna at $24 \mathrm{GHz}, 32 \mathrm{GHz}$, and $40 \mathrm{GHzof}$ both antenna ports. The simulated maximum gains are $2.8 \mathrm{dBi}$, $5.0 \mathrm{dBi}$, and $4.3 \mathrm{dBi}$ at the stated frequencies. Similarly, the simulated total efficiencies are $-0.8 \mathrm{~dB}(83 \%),-0.3 \mathrm{~dB}$ (93\%), and $-0.4 \mathrm{~dB}(91 \%)$.

As it can be observed, at $40 \mathrm{GHz}$ the directivity of the radiation pattern is not in the bore-sight (Z-direction, Theta $=0^{\circ}$ ) anymore but in Theta $= \pm 45^{\circ}$. This is caused by the currents in the ground plane, as the ground plane size is equal to $0.9 \lambda$ at the $40 \mathrm{GHz}$ operating frequency. Still, no deep zero is observed in the direction of boresight.

\section{CONCLUSIONS}

The paper presented a planar wideband dual-polarized patch antenna structure implemented on the PCB. The structure was studied with simulations and measurements in terms of $-10 \mathrm{~dB}$ impedance bandwidth. The simulated results predicted 24-40 GHz bandwidth against the 24.75-42.25 GHz measured ones.

The antenna structure was implemented on the ground plane of size $4.7 \times 4.7 \mathrm{~mm}^{2}$ which corners were cut out to improve XPD. Manufactured prototype antenna was measured and simulated with a $50 \Omega$ coaxial line feed. The simulated total efficiency and XPD were presented. The total efficiency predicted better than $-0.8 \mathrm{~dB}(83 \%)$ efficiency, whereas the XPD is better than $14 \mathrm{~dB}$ over the studied frequency bandwidth. Finally, the $3 \mathrm{D}$ radiation patterns of the dual-polarized patch antenna with both ports were presented at $24 \mathrm{GHz}, 32 \mathrm{GHz}$, and $40 \mathrm{GHz}$ with $2.8 \mathrm{dBi}$, $5.0 \mathrm{dBi}$, and $4.3 \mathrm{dBi}$ maximum gain.

\section{ACKNOWLEDGMENT}

This work has been supported by Academy of Finland project "Antenna Design at mm-Waves and Highly Directive Lens Antenna Simulations for sub-THz Band by Using Ray Tracing Platform" (grant no. 323857), and the National Natural Science Foundation of China project "mmWave Antenna Array Design for Wireless Channel Sounding" (grant no. 61911530771). The work is partly supported by the Academy of Finland project 6Genesis Flagship (grant no. 318927).

\section{REFERENCES}

[1] T. Rappaport, et al. "Millimeter Wave Mobile Communications for 5G Cellular: It will work!.", IEEE Access, Vol. 1, 2013, pp. 335-349.

[2] Z. Pi and F. Khan, "An Introduction to Millimeter-Wave Mobile Roadband Systems", IEEE Commuication. Magazine, Vol. 49, No. 6, June 2011, pp. 101-107.

[3] A. I. Sulyman, A. T. Nassar, M. K. Samimi, G. R. Maccartney, T. S. Rappaport, and A. Alsanie, "Radio Propagation Path Loss Models for $5 \mathrm{G}$ Cellular Networks in the $28 \mathrm{GHz}$ and $38 \mathrm{GHz}$ Millimeter-Wave Bands", IEEE Communication Magazine, Vol. 52, No. 9, September 2014, pp. 78-86.

[4] J. Yang and A. Kishk, "A Novel Low-Profile Compact Directional Ultra-Wideband Antenna: the Self-Grounded Bow-Tie Antenna", IEEE Transactions on Antennas and Propagation, Vol. 60, No. 3, March 2012, pp. 1214-1220.

[5] H. Raza, A. Hussain, J. Yang, and P.-S. Kildal, "Wideband Compact 4-Port Ddual Polarized Self-Grounded Bowtie Antenna", IEEE Transactionson Antennas and Propagation, vol. 62, no. 9, September 2014, pp. 4468-4473

[6] S. Mukherjee, A. Biswas, and K. V. Srivastava, "Broadband Substrate Integrated Waveguide Cavity-Backed Bow-Tie Slot Antenna", IEEE Antennas and Wireless Propagation Letters, Vol. 13, June 2014, pp. 1152-1155.

[7] S. M. Moghaddam, J. Yang, A. A. Glazunov, "Ultra-Wideband Millimeter-Wave Bowtie Antenna", International Symposium on Antennas and Propagation (ISAP), 30th Oct.-2nd Nov. 2017. 
[8] P. K. Panda, D. Ghosh, "Wideband bow-tie antenna with increased gain and directivityby using high impedance surface", Wiley International Journal of $R F$ and Microwave Computer-Aided Engineering, 3rd December 2018, 9 p.

[9] M- Sonkki, Z. Siddiqui, J. Chen, M. E. Leinonen, M. Berg, A. Pärssinen, "Study of Planar Wideband mm-Wave Bowtie Antennas Over PCB Ground Plane", $13^{\text {th }}$ European Conference on Antennas and Propagation (EuCAP), 31 $1^{\text {st }}$ March-5 ${ }^{\text {th }}$ April 2019, Krakow, Poland.

[10] Chun-Xu Mao, Steven Gao and Yi Wang, "Broadband High-Gain Beam-Scanning Antenna Array for Millimeter-Wave Applications", IEEE Transactions on Antennas and Propagation, vol. 65, no. 9, 2017, pp. 4864-4868.

[11] Qian Zhu, Kung Bo Ng, Chi Hou Chan and Kwai-Man Luk, "Substrate-integrated-waveguide-fed array antenna covering 57-71 $\mathrm{GHz}$ band for 5G applications", IEEE Transactions on Antennas and Propagation, vol. 65, no. 12, Jul. 2017, pp. 6298-306.

[12] In-June Hwang, Hye-Won Jo, ByungKuon Ahn, Ju-ik Oh and JongWon Yu, "Cavity-backed Stacked Patch Array Antenna with Dual Polarization for mmWave 5G Base Stations," 13th European Conference on Antennas and Propagation (EuCAP), 31st March-5th April 2019, Krakow, Poland. 